\title{
LA DERMATITE DES NAGEURS
}

\author{
Par C. DESPORTES
}

Il n'est pas exceptionnel d'observer en été - et surtout pendant les années de sécheresse - une affection cutanée éruptive et prurigineuse, chez les sujets qui ont plongé la totalité ou une partie de leur corps dans l'eau des rivières, des étangs, des mares et, d'une façon générale, dans toutes les collections d'eau douce un peu importantes : c'est à cette affection que l'on donne le nom de Dermatite des nageurs ou de Bouton caniculaire des baigneurs.

Les manifestations prurigineuses débutent presque toujours dans les premières minutes qui suivent le bain et c'est pourquoi les sujets atteints, sans connaîtne l'étiologie précise de la maladie, en comprennent rapidement la prophylaxie. L'évolution de la dermatite est, du reste, très favorable et, le plus souvent, le malade n'est pas amené à consulter. Cependant, cette éventualité peut se produire lorsque les manifestations deviennent intenses ou qu'elles se compliquent d'infections secondaires occasionnées par le grattage, mais alors, trop souvent, le diagnostic n'est pas porté et c'est Ia raison pour laquelle nous avons voulu attirer ici l'attention des médecins sur son existence en France.

La cause véritable de cette affection, déjà soupconnée en Suisse par Naegeli (1923) qui admet, pour la maladic désignée sous le nom de «Hundsblattern » (= éruption caniculaire), un «agent du règne animal », est bien établie par Cort (1928) au Michigan. Cet auteur montre, en effet, expérimentalement que la pénétration cutance des cercaires de certaines bilharzies (Trématodes), incapables d'évoluer ultérieurement chez l'homme, provoque chez lui une ćruption prurigineuse comparable aux dermatites observées chez les baigneurs du Douglas Lake.

Christenson et Greene (1928) font, quelques mois plus tard, les mêmes constatations, dans le Minnesota, pour la maladie nommée « swimmer's itch » et, à partir de 1930, une succession de publications va rapidement élargir les connaissances sur ce sujet et son étiologie parasitaire; ce sont les travatux de L. Szidat (1930) en Prusse Orientale; de C. Matherson (1930) et de E. Taylor et

Ans, de Pallasitologie, $\mathrm{T}, \mathrm{XX}, \mathrm{N}^{0 \mathrm{~s}}$ 5-6, 1944-1945, p. 263-278. 
H. Baylis (1930) à Cardifl ; de H. Vogel (1930), qui en fait une étude histo-pathologique expérimentale détaillée et lui identifie les cas signalés au Sud de Kiel; de E. Brumpt (1931), qui montre son existence en France, au Bois de Boulogne ; de F. Fülleborn (1932); de Szidat et R. Wigand (1934) ; de J. Mc Leod (1934) et die E. Swales (1936), au Canada; de G. La Rue (1935); ceux, très importants, de W. Cort et de S. Talbot, en 1936 ; de C. Barlow, démontrant le rôle analogue jouć par les cercaires des Bilharzies humaines (1938); de I. Buckley (1938), en Malaisie; d'E. Heber (1938); de Edwards et Prackett, elc...

Les recherches eflectuées par ces différents auteurs ont été plus spécialement entreprises au point de vue helminthologique et épidémiologique.

En France, la dermatitie des nageur's n'a donc été mentionnée que par E. Brumpt : c'est par une étude expérimentale approfondie qu'il put démontrer son existence. Mais cette affection est certainement beacoup plus répandue sur notre territoire: nous en faisons précisément connaìtre deux cas spontanés (dont une autoobservation) contractés en Indre-et-Loire.

Observations. - Pendant la période de sécheresse qui se prolongea de juin à octobre 1944 , les petites rivières étaient très basses et souvent même taries sur plusieurs kilomètres : ce fut notamment le cas du Mâble et de la Veude (afluents de la Vienne), pour lesquels il ne subsistait de l'eau que dans les bas-fonds devenus des mares résiduelles. Ces petites cuvettes constituaient le seul refuge possible pour la faune aquatique et la concentration en aninaux de toutes espèces y était très élevée : on y comptait, en particulier, par centaines ou par milliers les mollusques susceptibles de servir d'hòtes intermédiaires aux trématodes qui nous intéressent et, en fait, 12 p. 100 d'entre eux, spontanément infestés, émettaient des cercaires ocellées à queue bifide.

Pour effectuer des recherches scientifiques variées, nous sommes amenés à entrer dans l'une des mares résiduelles du Mâble et contractons une première fois la dermatite aux deux jambes le 24 aon̂t.

Les premiers phénomènes prurigineux sont remarqués environ cinq minutes après la sortie de l'eau; deux heures après seulement, apparaissent les premières manifestations objectives sous forme de macules très petites de 1 ou $2 \mathrm{~mm}$, et au nombre d'une centaine sur chaque jambe: elles s'arrêtent au niveau du genou, linite de l'immersion, mais épargnent Je pied, cependant accessible aux larves pendant le bain.

Dans la nuit (c'est-à-dire 8 heures environ après le début de l'infection), le prurit, beaucoup plus vif, provoque notre réveil, mais l'exposition des jambes à l'air apporte un soulagement. Ic jour suivant (12 heures après le début), l'éruption est très netle el représentéc par des éléments papu- 
leux, mais peu surélevés, de 5 a 10 mm. de diamètre, bien circonscrits, uniformément rouge-carminé, à peine atténués par la vitro-pression et très rarement centrés par un poil. La peau qui les entoure n'est pas modifiée: de teinte normale, elle n'est ni chaude, ni infiltrée. Jusqu'au quatrième jour, cette éruption ne se modifiera pas sensiblement, seule sa teinte s'assombrira progressivement en virant au violet. Pendant cette période, qui correspond à l'éruption papuleuse rouge, le prurit est intense, apparaissant par crises intermittentes de 20 minutes ou une demi-heure le jour, de façon presque continue la nuit, supprimant alors le sommeil pendant jlusieurs heures consécutives.

Pendant les crises, le prurit n'est pas uniformément étendu sur toute la zone infestée, mais il prédomine toujours à un instant donné sur une surface relativement réduite ( 2 à 3 éléments contigus), pour apparaître celui d'après sur une nouvelle zone souvent éloignée de la première : c'est ce déplacement continuel qui confère au prurit son caractère intolérable, et il devient finalement impossible de ne pas tenter un soulagement par grattage. Evitant autant que possible, dans ces conditions, l'infection secondaire, nous ne l'observons que pour l'un des éléments papuleux qui se change en une pustule assez large, à la face interne du genou droit.

La peau qui recouvre les papules reste assez ferme; le grattage ne provoque pas d'hémorragies. A partir du quatriène jour, une amèlioration sensible se fait sentir, le prurit est moins intense et disparaitra rapidement en mème temps que les éléments qui, semblant subir un desséchement profond, durcissent et prennent une teinte brunatre, puis s'éclairciront de la périphérie vers le centre jusqu'à leur effacement complet. Quelques-uns sont recouverts de squames épidermiques provoquées par le grattage. A aucun moment nous n'avons noté un cedème de la région ou une vésiculation des éléments; nous n'avons pas vu non plus dans notre cas la « réaction urticarienne initiale » de W. Cort, qui régresse une demi-heure après la pénétration des cercaires.

Nous contractons une seconde fois l'affection le 4 septembre, dans un bas-fond en contre-bas d'une écluse de la Veude. La dermatite est cette fois beaucoup plus importante, l'eruption tolale des deux jambes comprend, en effet, 700 éléments répartis très irrégulièrement et, pour la plupart, non centrés par un poil.

Par les caractères de son prurit, par l'évolution de ces éléments, cette seconde atteinte ne diffère en rien de la première; scule l'intensité du prurit est beaucoup plus élevée : en particulier pendant la seconde nuit, l'irritation est permanente; elle s'installe à o heures du soir, après le repas, pour ne s'atténuer que vers 5 heures du matin. A partir du quatrième jour, les manifestations prurigineuses régressent, comme la première fois, mais ici leur atténuation se fera plus lentement ; elles existent encore le soir du cinquième jour.

Nous avons naturellement tenté de découvrir un remede pour calner ces crises intolérables : parmi les différents produits a notre disposition, aucun des suivants n'apporte un soulagement efficace : pommade à l'oxyde 
de zine, pommade au titane, alcool, alcool-formol, teinture d'iode diluce, eat de Javel, vinaigre.

Mais, aussi bien avions-nous remarqué le rôle actif de la chalcur du lit ou du soleil dans la production des crises prurigineuses, aussi bien nous constations le bon effel du froid sur les parties atteintes: une vessie de

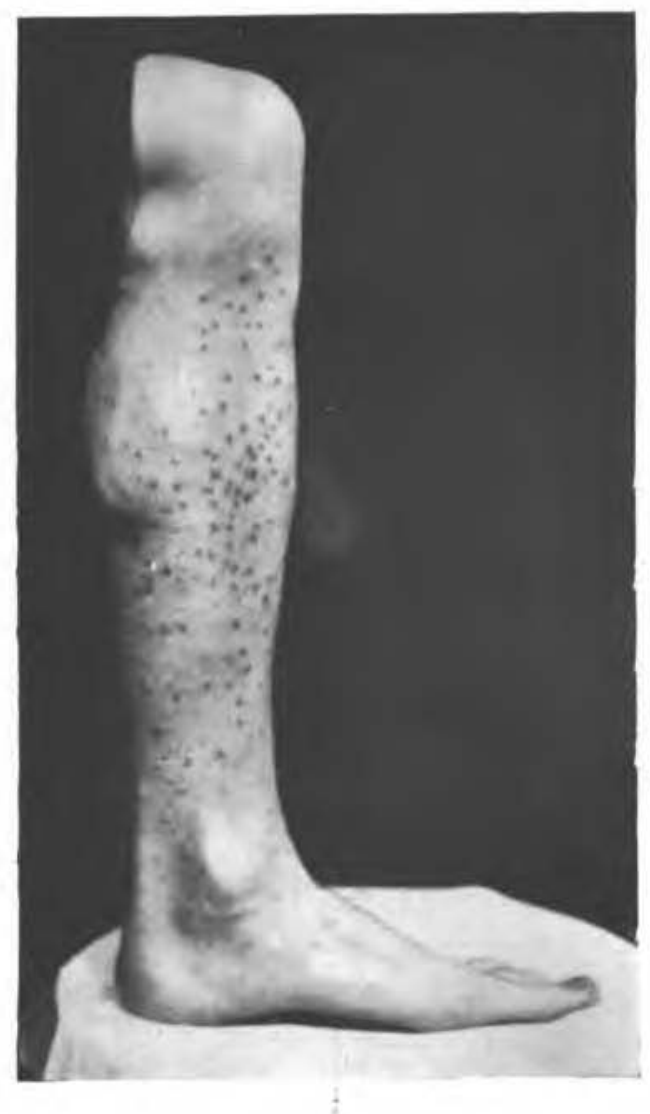

Fic, 1, - Dermalite des nageurs : cas spontané observé en Indre-et-Loire.

glace ou des vaporisations de chlorure d'éthyle, utilisées dès le début de la crise, l'arrètaient presque immédiatement; c'est ainsi que nous stoppons, au seuil de la troisième nuit, une crise qui aurait pu être très vive.

La seconde observation se rapporte à l'une de mes filles, alors âgée de 5 ans, qui, en m'accompagnant à la Veude, se contamine légèrement en plongeant les mains dans l'eau : un prurit assez vif ct quelques éléments 
papuleux apparaissent dans les 24 heures, pour s'estomper trois ou quatre jours plus tard.

A ces deux cas spontanés, nous devons ajouter l'observation suivante se rapportant a notre camarade Y. Campana, assistante de l'Institut de Parasitologie qui, au ccurs des récoltes effectuées par elle dans les mêmes mares, mais peut-être en d'autres points, ne se contamine ni la première ni la seconde fois. Pensant, sinon à une immunité, du moins à une nonréceptivité possible, nous tentons avec elle d'élucider ce point par l'expérimentation: une première application d'une vingtaine de furcocercaires ocellées contenues dans un couvercle de tube Borrel retourné sur la face interne de l'avant-bras gauche ne provoque aucun phénomène; une seconde application de 800 ou 1.000 cercaires déclenche, au contraire, en 10 minutes, un picotement assez vif et l'expérience est suspendue quelques minutes plus tard.

I,a surface de $45 \mathrm{~mm}$. de diamètre, exposée aux cercaires, prend rapidement une coloration rosée ; après 10 heures elle apparaît sous la forne d'une plaque rouge framboise surélevée, irrégulière par endroils, efiaçable à la vitro-pression ef formée par la confluence d'environ 200 éléments dont le centre sc verra beaucoup plus nettement le jour suivant: il existe en eflet, au point de pénétration de chaque cercaire, une vésiculctle de la taille d'une tête d'épingle contenant un liquide limpide ou opalescent. Jusqu'au cinquième jour, la coloration de fond reste assez vive et le prurit, quoique bien toléré (sans dloute parce qu'il est localisé en whe zone étroite), apparaît de temps à autre par de petites crises aiguës. Entre ces dernières subsiste une sensation de chaleur à peu près permanente et qui interdit le port d'une manche. Consécutivement au grattage inévitable, certaines vésicules se vident de leur contenu sans se reformer: toutes se dessèchent progressivement, laissant finalement au bout d'une semaine de petites acuminations rugueuses sur la plaque. Celle-ci vire, à son tour, à l'ocre clair, sans jamais avoir pris cette teinte violacée et soirbre constatée dans notre cas; elle ne s'affaissera complètement et ne perdra son aspect rugueux que beaucoup) plus tard.

On peut donc considérer que, dans cette expérience, sculement le quart ou le cinquième des cercaires utilisées, placées dans les meilleures conditions de pénétration possible, a perforé Ia peau. Nous ignorons évidemment en présence de combien de centaines de cercaires nous avions été personnellement exposé, mais de toute façon, et mêne par la seule symptonatologie, la réceptivité individuelle apparaît beaucoup plus faible dans la présente observation que dans les deux précédentes.

Il n'est pas sans importance de rappeler ici la curieuse remarque de Szidat el Wigand (1934), selon lacuelle les sujets blonds sont plus réceptifs que les bruns : elle semble confirmée dans les cas présents.

En conciliant ces observations aux travaux antérieurement publiés, nous croyons pouvoir grouper et résumer les connaissances actuelles de cette affection par l'étude suivante: 
Nom. - Dermatite ues Nagevrs, E. Brumpt, 1931.

Synonymes. - Bouton de canicule des baigneurs (1), P. Paris 1931. Schistosome dermatitis (2), W. Cort 1928 (U.S.A.). Swimmer's itch, in Christenson et Greene 1928 (U.S.A.). Cercarial dermatitis McLeod 1934 (U.S.A.). Slongh itch, in McLeod 1934 (U.S.A.). Hundsblattern, in Naegeli 1923 (Suisse). Aareausschlag (3), in Naegeli 1923 (Suisse). Exanthema caniculare Naegeli 1923. Zercarien-Dermatitis Vogel 1930 (Allem.).

? Kaburé (4), ficle Fülleborn 1932 (Jap.). Sawah itch, fide Buckley 1938 (Mal.).

Plusieurs de ces noms ont été utilisés par les habitants de certaines localités bien avant que la cause de la maladie fût établie ; leur identification est postérieure à 1928.

Mais, il est bien évident qu'une telle synonymie n'est acceptable que dans la mesure où l'on ne considère pas l'espèce à laquelle appartient la cercaire pathogène : elle reste intégralement valable pour la définition suivante :

Définition. - C'est une affection cutanée, estivale, éruptive, très prurigineuse, de courte durée, contractée par immersion en eau donce et due à la pénétration cutanée de Cercaires de trématodes non parasites de l'homme.

Il est bien évident que cette définition, dans son sens strict, n'est valable que pour la dermatite cercarienne, telle qu'elle sie présente en Europe et en Amérique du Nord, ou encore pour le «Sawah-

(i) Ce nom choisi par P. Paris (1931), et qui figure en tête d'une analyse des publications de E. Brumpt (1931), est en réalité la traduction de Hundsblattern (Hunds =: du chien, caniculaire: la (ianicule est en effet la période qui s'étend du 22 juillet au 23 aoùt et pendant laquelle, autrefois, Sirins (= Canicule), de la constellation du Grand-Chien, se couchait et se levait en même temps que le soleil. Sous l'effet de la précession des équinoxes, le lever héliaque de cette étoile se fait maintenant au délout d'aont ; Blattern $=$ boutons éruptifs).

(2) Ce nom a été domné par. Gort parce que les espéces recomnues pour agent de cette dermatile apparliennent à la grande famille des Schistosomida (dont le genre Schistosoma des bilharzioses humaines et animales est le type). Deux espèces de Strigeidea, un moment incriminces, semblent l'avoir été à tort (MfeLeod, 1934, 1936).

Comme le fail remalquer Fülleborn (1932), ce nom peut prêter à confusion, car, dans les bilharzioses humaines, on peut observer des phénomènes urticariens en rapport avec la migration des parasites ef leur developpement dans les vaisseaux hepatiques.

(3) = Eruption de I'Aar.

(4) Le Kaburé a été consicléré par quelques auteurs comme provoqué par la péncitration cutanée de Schisfosoma juponicum. Sans que la chose soit entièrement demonlée, on s'aceolede aujourd'lui pour en faire plutôt une termalile provoqué par des Cercaires de l'imatodes non humains. 
itch », provoquć par la cercaire de Schistosoma spindale, parasite du bétail.

Depuis les belles recherches de C. Barlow, on sait que des manifestations cutances analogues existent au début des bilharzioses humaines, c'est-à-dire consécutivement à la pénétration de cercaires de parasites qui nous sont propres, mais de telles manifestations ne représentent que le syndrome initial précurseur de manifestations viscérales beaucoup plus graves, c'est pourquoi on ne peut, même en donnant à la définition un sens plus large, assimiler de telles dermatites à la maladie qui nous occupe.

Historique. - Connue depuis longtemps par ses manifestations éruptives iet prurigineuses qui suivent le bain en eau douce, elle est rapportée à sa véritable origine par $W$. Cort, en 1928, aux EtatsUnis. Des études expérimentales entreprises en Amérique du Nord, en Angleterre, en Allemagne, en France et en Extrême-Orient en amplifient les connaissances.

En France, son existence est établie par E. Brumpt. Nous donnons ici les premières observations de cas spontanćs observés et contrôlés sur notre territoire.

Etiologie et pathogénie. - Nous avons dit que l'agent causal de l'aflection est la larve nommée Cercaire de certains Trématodes non parasites de l'homme. Pourquoi de telles larves, incapables d'achever leur évolution chez cet hôte, pénétrent-elles? Sans vouloir donner une solution - qui ne pourrait être que «finaliste»à ce problòme, nous nous devons de fournir ici quelques précisions :

Un grand nombre de trématodes, appelés * Trématodes digénétiques », admettent dans leur évolution deux générations : la première, formce d'une succession de stades larvaires, s'effectue chez un premier hòte qui, le plus souvent, est un mollusque aquatique; la seconde, représentée par des adultes, parasite les vertébrés.

Le stade cercaire constitue alors précisément la forme de passage du premier hôte au second et le passage peut se faire selon deux modes : ou bien la cercaire, libérée du mollusque, est ingérée par le vertébré (elle est alors très sourent enkystée sur une plante ou chez un nouvel animal avec lequel elle sera absorbee), ou bien elle nage à sa recherche; c'est ce qui se passie notamment pour les cercaires de la dermatite des nageurs. Or, une cercaire est un très petit animal : elle mesure à peine un demi-millimctre ; de plus, son existence est éphémère ( 48 ou $72 \mathrm{~h}$.) et si elle n'était pas douée d'un tropisme particulier qui l'oriente vers le vertébré, hôte définitif, il lui resterait très peu de chances pour assurer la conservation de 
son espèce... Ce tropisme est en général assez spécifique pour l'espece en cause, mais il n'est pas absolu et c'est pourquoi nous voyons des cercaires de trématodes de rongeurs ou d'oiseaux aquatiques s'égarer chez l'homme. Elles peuvent, du reste, aussi bien s'égarer et provoquer des dermatites chez d'autres hôtes (chien, cf. E. Heber', 1938) et l'homme ne représente ainsi (qu'un cas particulier.

Naturellement, les cercaires de beaucoup d'espèces ne sont pas attirées par l'homme et ne peuvent être incriminées comme agents de la dermatite, mais le nombre des especes, agents de l'infection, doit certainement être encore assez élevé. Comme la dótermination de ces larves est très délicate, seulement huit espèces, parmi celles considérées comme susceptibles de provoquer la maladie, ont pu être actuellement identifiées, et la forme adulte, de même que son hôte définitif, n'est connue que pour trois d'entre elles.

En France, l'espèce identifiée, au Bois de Boulogne, est Trichobilharzia ocellata, adulte chez le canard ( $\mathbf{E}$. Brumpt); elle admet la grande Limnée (Limnen stagnalis) pour hôte intermédiaire. Mais ce trématode n'est certainement pas le seul qui puisse êtrie en cause dans notre pays, puisque certaines des cercaires reconnues pour pathogènes en Indre-et-Loire ne se développent pas expérimentalement chez le canard. En outre, nous leur connaissons au moins deux hôtes intermédiaires : Limnea stagnalis et L. auricularia, dont le second seul étail présent dans les mares du Mâble.

Le rôle joué dans la production de la maladie par les différentes espèces est du reste à peu près le même (W. Cort, 1936, a) et l'affection est incontestablement due au seul effet de leur pénétration : les eaux polluées et riches en bactéries ne semblent pas modifier sá symptomatologie; tout au plus, peut-on admettre avec Cort que l'abondance des germes bactériens en suspension favorise, par son ensemencement au cours du grattage, la formation des éléments pustuleux. Interprétant les modifications histologiques des tissus, traversés par les cercaires, Vogel considère que les accidents sont provoqués par les sécrétions toxiques, histolytiques des glandes de pénétration des parasites. Ceux-ci doivent du reste succomber et être détruits par le tissu réactionnel. On considère ainsi les phénomènes, observés au cours de la dermatite, comme étant la traduction d'une réaction de défense ou même une immunité naturelle de l'homme à un parasite qui ne lui est pas spécifique (Vogel, 1930). A l'appui de cette conception, C. Barlow souligne que la pénétration des cercaires de schistosomes, agents de bilharzioses humaines, paraît plus nette et plus fréquente chez les sujets infes- 
tés depuis longtemps et qui, de ce fait, auraient acquis une certaine immunité.

Les cercaires perforent facilement l'épiderme et leur pénétration au niveau des poils, si elle existe (Mc Leod, 1934), est exceptionnelle (auto-biopsie de Vogel). Cette pénétration s'effectue cerlainement en moins de dix minutes (E. Brumpt, 1931). Selon l'espèce, ces larres se tiennent en suspension dans l'eau ou groupées par milliers à sa surface (ce qui explique l'intensité de certaines affections), mais, de même que les mollusques qui les libèrent, elles nagent plus volontiers au voisinage des plantes aquatiques, des branches et des objets immergés ou des berges.

Répartition géographique. - France : Paris (Bois de Boulogne). Indre-et-Loire (Richelieu).

? Nièvre (1).

Angleterre : Cardiff.

Suisse : Lac de Constance; Aar.

Allemagne : Prusse orientale ; Holstein.

Etats-L'nis : Michigan, Minnesota, Wisconsin, Iowa, elc.

Canada : Manitoba, Saskatchewan.

Haïti :

Malaisie :

? Extrême-Orient (《 Kabure »).

Saison de prédilection. - C'est une affection de l'été (« Hundsblattern ») : elle disparait avec les premiers froids en même temps que s'arrêtent les émissions de cercaires par les mollusques qui, eux-mêmes, se cachent.

Les années de sécheresse favorisent l'aflection en tarissant les eaux et provoquant la concentration des mollusques.

Age, sexe, profession. - Les enfants et les femmes seraient plus sensibles que les hommes (Mc Leod) et les blonds plus réceptifs que les bruns (Szidat et Wigand). Mc Leod considère qu'environ une personne sur six semble réfractaire à cette maladie. En réalité, tous les intermćdiaires entre la réceptivité et la non-réceptivité existent : certains sujets ressentent parfaitement le picotement initial qui indique la pénétration des cercaires et ne font pas

(1) Cette localité a été donnée par Cort consécutivement à une lettre reçue dans laquelle on l'informe que des soldats américains auraient contracté, après s'être baignés dans une jivière de la Niève, une affection comparable à celle qu'il a décrite. 
ultérieurement de dermatite iet d'autres ne sont même pas aftaqués par les cercaires pathogènes.

\section{Symptomatologie}

Incubation. - Le prurit succède presque immédiatement au picotement qui accompagne la pénétration des cercaires, on ne peut donc pas parler d'une incubation.

Invasion. - $1^{\circ}$ Dans la forme spontanée habituelle, elle n'est représentée que par le prurit et les macules localisées sur les surfaces exposées à l'infestation.

Le pruril est ressenti très précocement, à la sortie de l'eau ; il est généralisé sur les surfaces contaminées, net et encore tolérable à ce stade.

Les macules n'apparaissent qu'à la fin de la première ou de la deuxième heure qui suit la contamination, sous forme de petites taches régulières, arrondies, de 1 ou $2 \mathrm{~mm}$. de diamètre, rouges et qui s'effacent à la vitro-pression; la peau qui les environne reste normale. Ces éléments sont, lorsque l'immersion est totale, plus abondants sur les membres que sur le trone; les mains et les pieds sont moins atteints que les poignets ou les jambes. Christenson et Greene (1928) remarquent leur concentration sur les régions pileuses.

$2^{\circ}$ Dans les infestations intenses et concentrées (infestations expérimentales); chez des sujets particulièrement sensibles, le prurit initial peut s'accompagner d'une éruption de type urticarien qui, tantôt est de courte durée (Cort 1928), tantôt est permanente, formant un placard rouge, au milieu duquel les points de pénétration apparaissent sous l'aspect de très petites macules de teinte plus vive (E. Brumpt, 1931).

Etat. - Cette période est en général atteinte en dix ou douze heures. Elle est essenticllement caractérisée par le prurit intense et les papules, accessoirement par l'odème et les troubles généraux.

Prurit. - Localisé aux surfaces afteintes, il apparaît sous forme de crises, et dure cinq jours environ.

Le jour, les crises sont irrégulièrement réparties, provoquées par la chaleur solaire, le port des vêtements et les attouchements : elles ont une durée de 20 ou 30 minutes.

La nuit, elles sont très vives et prolongées : elles réveillent le malade lorsqu'elles apparaissent pendant le sommeil et entraînent l'insomnie. Le contact du drap est insupportable. 
Pendant la crise, l'irritation passe d'un petit groupe d'éléments à un autre, souvent distant, à des instants très rapprochés : cette mobilité contribue à le rendre intolérable.

Papnles. - Eiles succèdent aux macules et évoluent toutes synchroniquement (sauf lorsqu'il y a réinfestation, chaque contamination formant de nouveaux groupes d'éléments).

Mesurant de 6 à $15 \mathrm{~mm}$. de diamètre, elles sont arrondies, bien circonscrites, peu élevées, lisses, fermes et d'abord de teinte uniformément rouge-carminé, sauf au centre où il existe souvent une puncture hémorragique, plus rarcment et seulement chez certains sujets une vésiculette hyaline ou opalescente, mais presque jamais un poil. Ces éléments s'effacent mal à ]a vitro-pression.

A partir du troisième jour, leur teinte vire au violet et elles ne s'effacent plus à la pression du verre. Elles régressent vers le septième jour en séchant, durcissant et en prenant une teinte brunâtre; quelquefois elles sont recouvertes de squames épidermieques, plus spécialement lorsqu'elles ćtaient primitivement centrées par les vésiculettes.

Les papules sont en général assez fermes et le grattage ne détermine pas d'hémorragies, mais il peut provoquer, par infection secondaire, leur transformation en pustules.

OEdème. - Il n'apparaît que dans les cas graves et seulement chez certains sujets particulièrement réceptifs.

Troubles généraux. - Ils n'existent pas le plus souvent : on a pu noter, cependant, un état de prostration accusé au cours de certaines dermatites cercariennes. L’insomnie est par contre très fréquente. La maladie n'est pas fébrile.

Terminaison. - Elle a lieu en général le cinquième ou sixième jour avec la disparition du prurit et la régression de l'éruption : la zone pigmentaire des cléments peut cependant encore s'observer après quinze jours.

Evolution et pronostic. - Dans tous les cas simples, ils sont très favorables, la dermatite des nageurs guérissant spontanément.

Immunité. - Il n'y a pas d'immunité ou du moins, si elle existe, s'ćtablit-elle extrêmement lentement (Cort, 1936).

Complications. - Elles sont dues à l'infection secondaire qui peut prolonger l'affection plusieurs semaines.

\section{Formes GliniQues}

La maladie ne diffère pas selon l'espèce à laquelle appartient la cercaire, ni selon la région où on l'observe, l'âge, le sexe ou le siège, mais nous devons distinguer : 
$1^{\circ}$ Selon la réceptivité des sujets :

la forme avec incubation : chez certains sujets, sans doute parce qu'ils sont peu sensibles au prurit et peu réceptifs à la maladie, les premiers phénomènes irritatifs, puis éruptifs, apparaissent seulement au bout de plusieurs heures (Naegeli en signale deux cas, et la même l’emarque aurait été faite chez plusieurs sujets à Haïti, mais rien ne prouve qu'il ne s'agissait pas aussi bien d'une autre maladie) ;

la forme des Blonds, plus grave que celle des Bruns (Szidat et Wigand).

$2^{\circ}$ Selon l'éruption :

la forme papulense simple, classique;

la forme papulo-vésiculeuse, dans laquelle chaque papule est centrée par une vésiculette;

la forme pustuleuse, lue à l'infection secondaire;

la forme à éléments d'àge varié. Une première infestation ne conférant pas l'immunité, un même sujet, contaminé à des intervalles rapprochés, peut présenter des groupes d'éléments d'âges différents.

3* Selon la concentration des germes :

la forme « des volontaires ». Nous n'hésitons pas à donner ce nom à la forme particulière que prend l'affection expérimentale, du fait de la concentration des larves actives sur une surface limitée de la peau : la littérature en comprend déjà une vingtaine de cas et il y en aura certainement d'autres puisque bien des cercaires incriminées restent à identifier (Szidat 1942), et par conséquent à expérimenter sur des volontaires.

Chez elle, le picotement à la pénétration des trématodes est toujours ressenti.

La première heure est souvent marquée par l'apparition d'un placard éruptif urticarien, qui peut s'effacer ou au contraire subsister plusieurs jours comme « teinte de fond $»$ il épouse alors souvent le contour du récipient contenant les larves et appliqué sur la peau.

Les éléments eux-mêmes sont le plus souvent confluents et souvent leur centre porte une vésiculette.

L'œdème apparaît également presque toujours dans ces formes. Enfin, la zone prend un aspect râpeux à la terminaison de la maladie

Histologie pathologique. - Les modifications histologiques sont partiellement connues par l'examen des biopsies que Vogel et Mc Leod pratiquèrent sur eux-mêmes après s'être infectés expérimentalement.

Pour Vogel, la cercaire, après avoir perforć l'épiderme, s'ache- 
mine d'abord parallèlement à sa surface, entre les cellules, puis s'enfonce en profondeur, traçant ainsi un canal de $1 \mathrm{~mm}$. environ qui s'étend jusqu'au derme. Les parois du canal sont formées de cellules hypertrophićes vacuolaires, à noyau rétracté, séparées les unes des autres par des infiltrations liquides, et sa lumière contient elle-même des cellules détruites; polỹnucléaires et lymphocytes très abondants.

Mc Leod, au contraire, écrit que la pénétration s'effectue au niveau des follicules pileux; la larve gagnerait ensuite, par cytolyse, le tissu sous-cutané qu'elle détruirait à distance par ses sécrétions. L'espace apparaìt infiltré et le tissu environnant envahi par les leucocytes.

Cette divergence entre les vues de Mc Leod et celles de Vogel, qui semblent plus conformes à ce que nous avons pu observer (absencie de poil au centre de l'élément), peut tenir aux cercaires utilisées.

Diagnostic. - Il est en général très facile et repose sur :

- Ia notion saisonnière;

- la notion de contamination par l'eau;

- le prurit intense;

- l'éruption maculo-papuleuse dont les éléments sont tous au même stade (penser, en cas contraire, aux réinfestations possibles).

Diagnostic différentiel. - Dans la pratique, il ne se pose pas puisque les accidents surviennent immédiatement après le bain et l'on ne peut pas confondre cette maladie :

- au début, avec l'urticaire, dont la bulle siège sur un fond érythémateux et disparaît sans laisser de traces;

- à la période de déclin, lorsque le prurit a disparu, avec :

- le lichen-plan, évoluant par poussées et s'accompagnant de lésions des muqueuses;

- les syphilides papuleuses, à évolution lente, de teinte cuivrée, entourées de la classique collerette de Biet.

Jans les formes impétiginisées, elle peut naturellement simuler toutes les pyodermites banales, dont elle ne représente en somme qu'un cas particulier. Elle se distingue cependant aisément de :

- la folliculite disséminée du thorax, dite "des piscines», dont les éléments pustuleux sont centrés par un poil :

- la gale (1) infectée, dont les éléments sont accompagnés de sillons et de vésicules perlées.

(1) On retrouve dans « Swimmer's itch 》 (= gale des nageurs) un rappel de cette analogic entre les deux affections. 
Il est beaucoup plus difficile de la distinguer, sans recourir à l'interrogatoire, des piqùres de moustiques concentrées, et surtout de la dermatite produite par la perforation des larves d'ankylostome ou «water-itch », dans les pays où les deux affections coexistent. D'après F. Fülleborn (1932), la confusion aurait été faite chez les sujets travaillant dans les rizières. En Afrique, en Amérique Centrale et peut-être en Extrême-Orient, on songera toujours à un début de bilharziose.

Traitement. - Les médicaments usuels destinés à calmer le prurit semblent n'avoir aucun effet favorable dans les cas importants. Par contre, la cryothérapie est riemarquable. On peut utiliser la vessie de glace ou les vaporisations de chlorure d'éthyle : il suffit alors de promener le jet sur quelques zones successivement prurigineuses pour faire disparaître la crise entic̀re.

Il est difficile d'éviter l'infection secondaire autrement que par des lavages fréquents de la peau à l'eau savonneuse et froide, le port d'un linge étant presque toujours intolérable.

Prophylaxie individuelle. - On évitera naturellement de retourner dans les eaux infectées ou l'on portera des bottes et des vêtements étanches.

Prophylaxie générale. - Lorsque la chose est possible et nécessaire, on tentera de supprimer les hòtes intermédiaires (mollusques) de ces trématodes. Le sulfate de cuivre (à 1: 500.000) a donné des résultats très satisfaisants à la Station de balnéation près de Cardiff. Le faucardage contribue également à éloigner les mollusques; en immergeant alors des faisceaux de branches ou de plantes, on peut capturier en quelques jours un grand nombre de mollusques. Lorsqu'il est réalisable, le tarissement complet de la Station est à préconiser, mais il ne faut pas oublier que sur les fonds boueux les mollusques résistent parfaitement pendant plusieurs semaines à une dessiccation qui semble complète.

L'utilisation des animaux destructeurs de mollusques, les canards en particulier, est une arme à double tranchant : ils peuvent en effet servir d'hôtes définitifs aux trématodes et en favorisent alors la dissémination.

\section{RÉSUMÉ}

Nous rapportons les observations de cas spontanés et expérimentaux de Dermatite des nageurs, contractée en France, puis, faisant une revision des travaux publiés sur cette affection, nous donnons 
de la maladie une étude générale : synonyunie, définition, historique, éliologie et pathogénie, répartition géographique, symptomatologie, formes cliniques, histologie pathologique, diagnostic, prophylaxie individuelle et génćrale. Nous notons le bon effet de la cryothérapie.

\section{Bibliographie}

Banow (C. H.). - Is there dermatitis in Egyptian schistosomiasis ? Amer. $J$. Hyg., XX1V, 1936, p. 587-599.

Bhunp' (E.). - Prurit et dermatites produits chez les nageurs par des cereaires de mollusques d'eau douce. C.R. Ac. Sc., CXCIII, 1931, p. 253-255.

- Cercaria ocellata, déterminant la dermatite des nageurs, provient d'une bilharzie des canards. C.R. Ac. Sc., CXCIII, 1931, p. 612-614.

Buckicy (J. J. C.). - On a Dermatitis in Malays caused by the Cercarine of Schistosoma spindale Montgomery 1906. J. Helminth., XVI, 1938, p. 117-120.

Chustexson (R. O.) et Gresere (W. P.). - Studies on biological and medical aspects of Swimmer's itch. Schistosome dermatitis in Minnesota. Minnesota lled., XI, 1928, p. 573-575.

Con (W. W.). - Schistosome dermatitis in the Inited States (Michigan). J. Amer. Med. Ass., Xc, p. 1027-1029.

a) Studies on Schistosome dermatitis. I. Present status of the subject. Amer. I. HIJg., XXIII, 1936, p. 3+9-371.

b) Studies on Schistosome dermatitis. IV. Further information on distribution in Canada and the L'nited States. Amer, J. Hyg., XXIV, 1936, p. 318-333.

Livalids (A. C.) et Bracketr (S.). - A « Swimmer's itch 》, Schistosome dermatitis. W'isconsin Med. Jour., XXXVII, 1938, p. 543-5+7.

FülLEbors (F.). - Haut und Helminthen. Handbuch der Haut-und Geschlechtskrankheiten. Berlin, 19:2, p. 708-800.

Heriben (E. C.). - Schistosome dermatitis in dogs. J. Parasit, XXIV, 1938, p. $474-475$.

LA Rue (G. R.). - Schistosome dermatitis. Mich. Publie Health, XXIII, 1935, p. $87-90$.

Mathesox (C.). - Notes on Cercaria elew Miller as the probable cause of an outbreak of dermatitis at Cardiff. Trans. Koy. Sor, Trop. Ifed. Hyg., XXIII, 1930, p. 121-424.

MeLizon (J. A.). - Notes on erearial dermatitis with descriptions of the causative organisms, Cercaria wardlei n. sp., Cercaria bajkovi n. sp. and the parthenogenetic stage of Cercaria elve Miller. Canad. J. Res., X, 1934, p. $394-403$.

Nabgrat. - Ucber einen beim Baden entstehenden Hautausschlag, die sog. Hundsblattern (Exanthema caniculare). Schweiz. med. Wochenschr., $\mathrm{N}^{\circ} 49,1923$, p. 1121-1122.

Panis (P.). - Boutons de canicule des baigneurs. Bull. Sc. Bourgogne, I, 1931, p. 86-87.

Swales (W. E.). - Schistosome dermatitis in Canada. Noles on two causative agents and their snail hosts in Manitola. Canat. J, Res., XIV, 1936, p. $6-10$. 
Szidat (L.). - Hautinfektion bei Parasiten inslesondere bei Bilharziella polonica Kow. Arch. f. Derm. u. Syphilis, CLX, 1930, p. 304-308.

- Was ist Cercoria ocellata ln Valette? Morphologie und entwicklungsgesshichtliche Untersuchungen über der Erreger der europäischen Cercarien-Dermatite des Menschen. Dents. Tropenmed. Zeitsch., XLVI, 1942, p. 481-497, 509-524.

SzIDAT (L.) et WIGAND (R.), - Leitfaden der einheimischen Wurmkrankheiten des Menschen, Leipzig, 1934, 212' pp.

Talbot (S. B.). - Studies on Schistosome dermatitis. II. Morphological and life history studies on three dermatitis-producing schistosome cercaria, C. elva Miller, C. stagnicola n. sp. and C. physella n. sp. Amer. Journ. Hyg., XXIII, 1936, p. 372-384.

Taylon (E. L.) et Baylus ( $H, A$.$) . - Observations and experiments on a derma-$ titis-producing Cercaria and on another Cercaria from Limnea stagnalis in Great Britain. Trans. Roy. Soc. Trop. Med. and Hyg., XXIV, 1930, p. 219-244.

Vogel (H.). - Hautveranderungen duch Cercaria ocellata. Dermatol. Wochenschr., $\mathrm{XC}, \mathbf{1 9 3 0 , \mathrm { p }} \mathbf{5 7 7 - 5 8 1 .}$

Cercarien-Dermatitis in Deutschland. Klin. Wochenschr., IX, 1930, p. 883886.

Institut de parasitologie de la Facnlté de médecine de Paris.

Station expérimentale de Richelien (Indre-et-Loire).

(Directeur : Prof. E. Brumpt). 